\title{
High Grade Uterine Sarcoma with Concurrent Metastatic Adeno- carcinoma to the Uterus: A Case Report
}

\author{
Luke King ${ }^{1}$, Radhika P Gogoi ${ }^{2}$, Christopher Hummel ${ }^{2}$, Zongming Chen ${ }^{3}$ and Ashlee Smith ${ }^{2}$ \\ ${ }^{1}$ Department of Obstetrics \& Gynecology, Geisinger Medical Center, Danville, PA, USA \\ ${ }^{2}$ Department of Gynecologic Oncology, Geisinger Medical Center, Danville, PA, USA \\ ${ }^{3}$ Department of Pathology, Geisinger Medical Center, Danville, PA, USA
}

*Corresponding author: Luke King, Department of Obstetrics \& Gynecology, Geisinger Medical Center, Danville, PA, USA, E-mail: ljking1@geisinger.edu

\section{Background}

Uterine cancer remains a relatively common cancer among women accounting for $3.6 \%$ of all new cancer cases in the United States [1]. Uterine sarcomas are a rare subset of uterine malignancies arising from mesenchymal origin and account for 3 to $7 \%$ of all uterine cancers [2]. Sarcomas possess a diverse array of clinical and histopathologic features which can make it difficult to delineate between the various classifications including: Leiomyosarcoma, endometrial stromal sarcoma, undifferentiated endometrial sarcoma, and smooth muscle tumors of uncertain malignant potential (STUMP). In an attempt to better classify sarcomas based on their different biologic behaviors, the International Federation of Gynecology and Obstetrics (FIGO) recently revised the criteria for classification and staging of uterine sarcomas [3].

Given the rarity of uterine sarcomas, there is a limited number of published reports describing high grade uterine sarcoma diagnosed concomitantly with a separate metastatic cancer to the uterus. We provide a unique case of high grade uterine sarcoma with concurrent metastatic poorly differentiated adenocarcinoma with focal signet ring cell features.

\section{Case Report}

A 59-year-old G1P1001 with a past medical history of hypertension, benign thyroid nodules, class III obesity (body mass index $35.02 \mathrm{~kg} / \mathrm{m}^{2}$ ) and appendecto- my with colonic resection due to intussusception as a child, presented for gynecologic consultation for a large uterine mass found on transvaginal ultrasound following a 2-month history of lower abdominal pain, vaginal bleeding, and malodorous vaginal discharge.

An endometrial curettage showed atypical cells with stromal proliferation concerning for malignancy. A follow-up computed tomography scan of the abdomen and pelvis revealed an enlarged heterogeneous uterus measuring approximately $20 \times 10 \times 13 \mathrm{~cm}$, omental caking, and abdominopelvic ascites (Figure 1 ). After receiving informed consent, patient was scheduled for an exploratory laparotomy, removal of pelvic mass, total abdominal hysterectomy, bilateral salpingo-opherectomy, and tumor debulking. Preoperative CA-125 measured $368.9 \mathrm{U} / \mathrm{mL}$.

Prior to scheduled surgery, the patient developed worsening vaginal bleeding that required immediate surgical management. Intra-operative examination revealed a significantly enlarged uterus secondary to an intrauterine mass with suspected involvement of the serosa and bilateral ovaries. The abdominal cavity contained a large amount of ascites and diffuse carcinomatosis to include omental caking, extensive diaphragmatic involvement, and diffuse mesenteric and small bowel lesions. The rectosigmoid was densely adhered to the adnexa and posterior uterus. The patient was suboptimally debulked and underwent a supracervical hysterectomy with residual disease.

Citation: King L, Gogoi RP, Hummel C, Chen Z, Smith A (2018) High Grade Uterine Sarcoma with Concurrent Metastatic Adenocarcinoma to the Uterus: A Case Report. Obstet Gynecol Cases Rev 5:127. doi.org/10.23937/2377-9004/1410127

Accepted: July 10, 2018: Published: July 12, 2018

Copyright: (C) 2018 King L, et al. This is an open-access article distributed under the terms of the Creative Commons Attribution License, which permits unrestricted use, distribution, and reproduction in any medium, provided the original author and source are credited. 
Specimens sent for pathologic studies included uterus, bilateral tubes and ovaries, and portion of omentum. Gross examination of the uterus demonstrated a $10.5 \times 10.4 \times 4.5 \mathrm{~cm}$ mass occupying $80 \%$ of the uterus. The mass bordered, but did not invade, the serosa.

Microscopic examination of the uterus exhibited areas of both low-grade and high-grade sarcoma (Figure 2). Focal vascular invasion by low grade tumor was

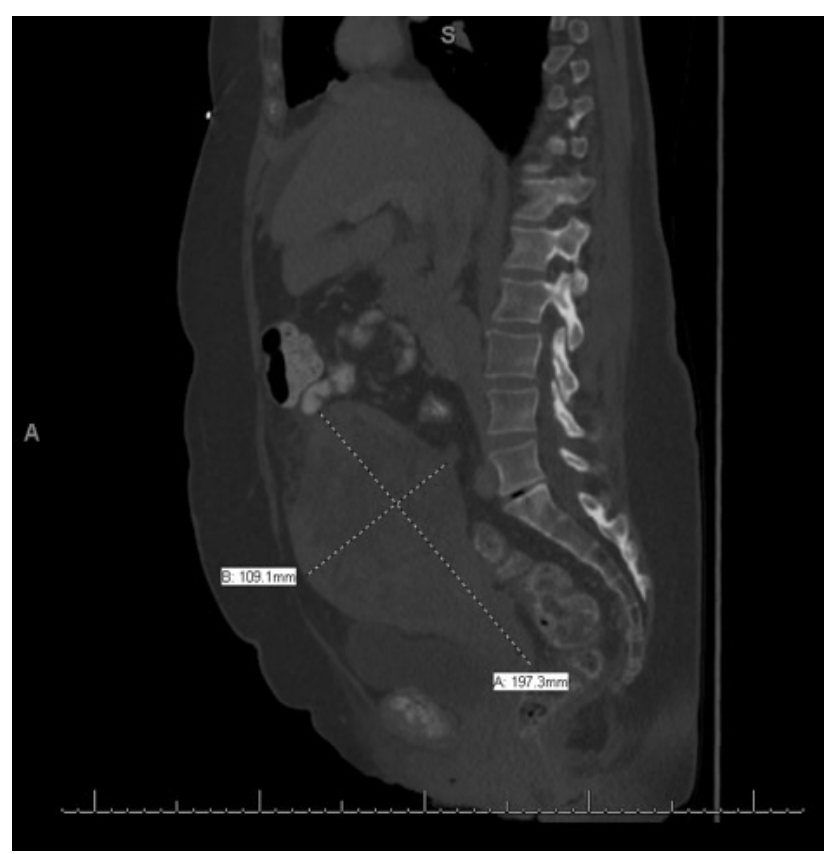

Figure 1: Computerized tomography of the abdomen and pelvis displaying an enlarged uterus. identified. The tumor appeared to extend to the cervical margin. Immunostains were performed on the uterine tissue. The low-grade tumor cells were positive for CD10 and negative for Caldesmon. In the high-grade tumor area, CD10 expression was diminished with patchy Cyclin D1 positivity and focally positive areas of Caldesmon. A focus of leiomyoma was also noted.

Microscopic analysis of the omentum, bilateral tubes and ovaries revealed metastatic poorly differentiated adenocarcinoma favoring an intestinal origin (Figure 3). Sections of the omentum showed poorly differentiated adenocarcinoma cells with mucinous features and signet ring cells. Immunostains of these tumor cells were

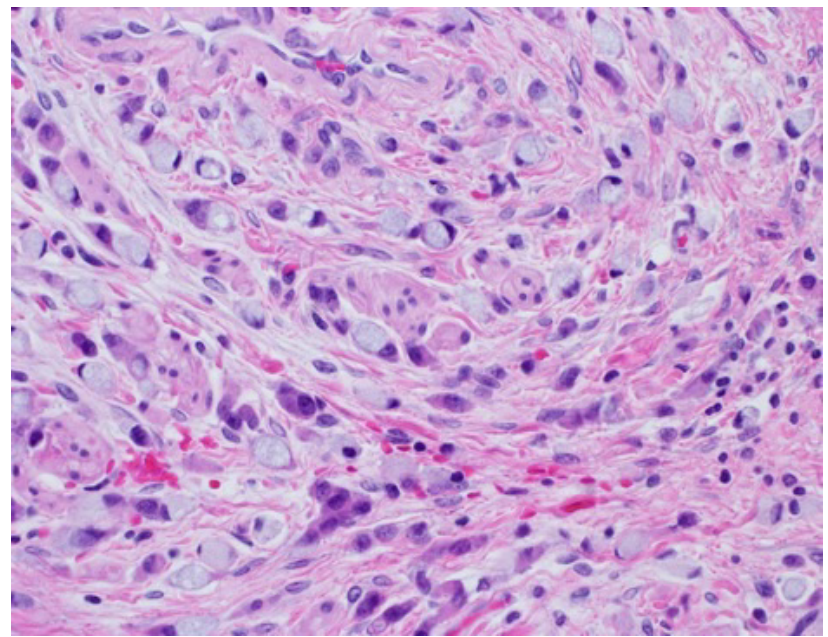

Figure 3: Metastatic poorly differentiated adenocarcinoma with focal signet ring cell features stained with H\&E at 40x.

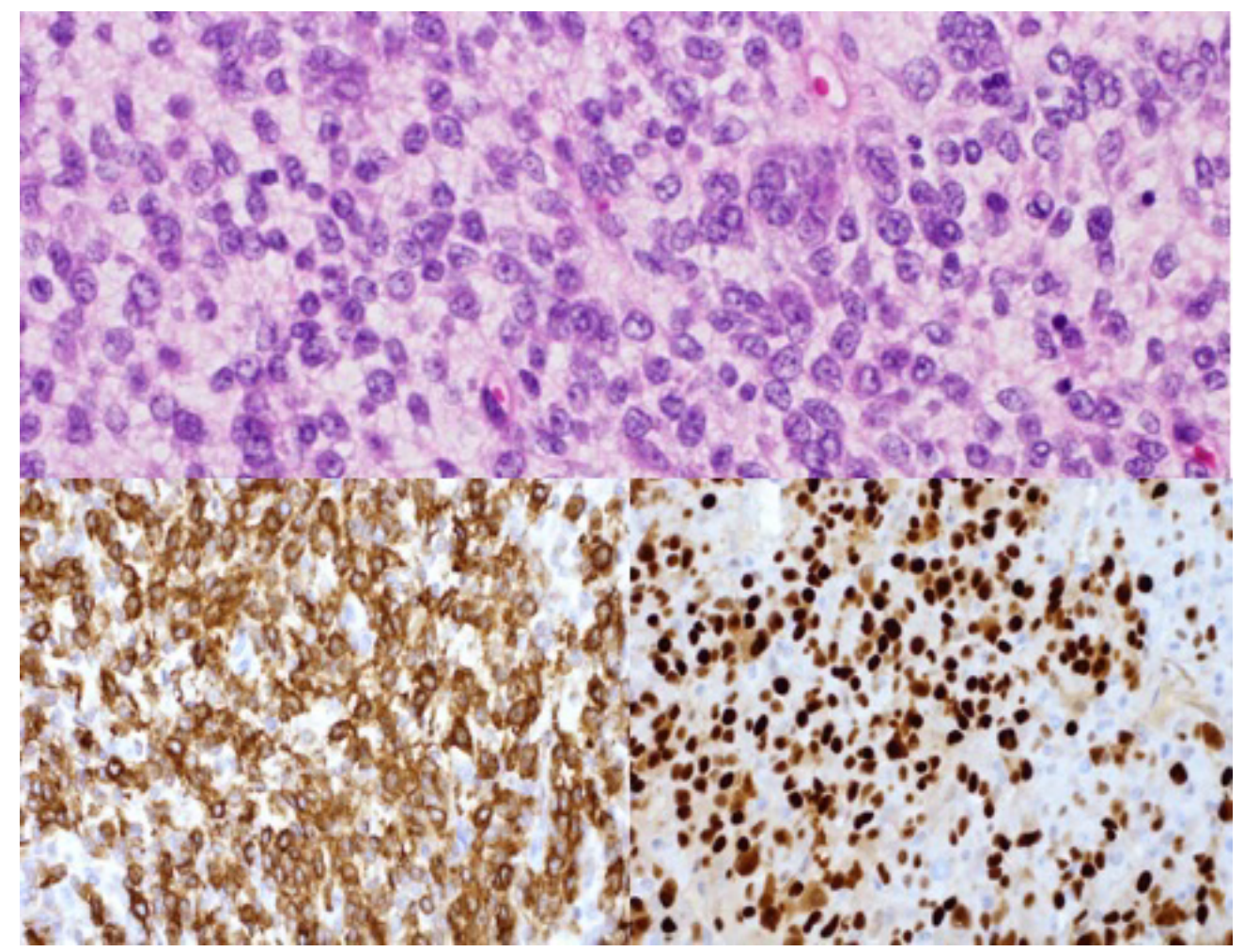

Figure 2: Uterine sarcoma tissue at 40x.

The top image illustrates high grade uterine sarcoma with H\&E staining. High grade cancer tissue stained positive for marker CD 10 (bottom left) and Cyclin D (bottom right). 
positive for CK20, CDX2; negative for CK7, GATA 3, TTF1 , and Synaptophysin. Immunohistochemical (IHC) detection of mismatch repair proteins displayed normal expression of MLH-1, MLH-2, MSH-6 and PMS2 in the adenocarcinoma tissue. KRAS and BRAF mutation testing was negative.

The original pathology report supports a diagnosis of Stage IB endometrial stromal sarcoma of the uterus and metastatic poorly differentiated adenocarcinoma likely an intestinal origin. An outside consultation at the John Hopkins Medical Institutions confirmed the metastatic disease as poorly differentiated adenocarcinoma with focal signet ring cell features. Pathologic review of the uterine mass, however, was interpreted as high grade sarcoma consistent with epithelioid leiomyosarcoma.

The origin of the primary adenocarcinoma remains unknown. The patient previously received a colonoscopy within two months of this diagnosis that found a benign $12 \mathrm{~mm}$ sessile polyp in the sigmoid colon. A post-operative upper endoscopic study was unremarkable. A positron emission tomography-computed tomography revealed metabolically active para-aortic lymph nodes, left femoral lymph nodes, and cervical mass with tumor thrombus involving the IVC, iliac veins, and left gonadal vein.

This case was presented at both the Gynecologic Oncology and Gastroenterology multidisciplinary meetings. Patient was amenable to receiving anastrozole due to positive tumor expression of the estrogen receptor and to begin a biweekly combination chemotherapy regimen of FOLFOX 6, consisting of leucovorin calcium, 5 -fluorouracil, and oxaliplatin. Patient is currently alive and undergoing treatment.

\section{Discussion}

Uterine leiomyosarcoma (ULMS) and endometrial stromal sarcoma (ESS) are the most common mesenchymal tumors of the uterus accounting for $30 \%$ and $10 \%$ of all uterine sarcomas, respectively [4,5]. Hysterectomy is the cornerstone for treatment for high grade uterine sarcomas. Due to the limited data supporting adjuvant radiation or chemotherapy treatment, options for adjuvant therapy remain controversial [6].

The majority of ULMS cases are diagnosed in the early stages. However, due to the aggressive behavior and high rate of recurrence, the diagnosis ULMS continues to carry a poor prognosis. Over $65 \%$ of women treated for ULMS will develop recurrent disease and less than $15 \%$ of women survive beyond 5-years post-relapse [7]. In comparison, ESS has a 5-year disease-specific survival rate of $76.2 \%$ [8]. Despite having a favorable survival rate, up to $76 \%$ of stage III-IV ESS will recur within 10 years of diagnosis [9].

The histopathological classification of the uterine sarcomas remains extremely difficult, particularly in cases of high grade disease. IHC markers aid in the diagnosis and are crucial to delineate between the various high grade uterine sarcomas that share similar morphologic features. ULMS often displays positive expression of smooth muscle markers (Smooth Muscle Actin, Desmin and $\mathrm{H}$-Caldesmon) as well as hormone receptors (progesterone and estrogen receptors). High grade ESS, on the other hand, demonstrates immunoreactivity to CD10, Cyclin D1 and BCOR markers [10-12].

In our case, the tissue containing high grade sarcoma displayed focal expression of CD10 and Cyclin D1, favoring a diagnosis of ESS, but lacked BCOR. In support of a diagnosis of ULMS, the tumor tissue contained focally positive areas of Caldesmon, a smooth muscle marker, but was negative for Desmin. There is evidence in the literature, however, to support that epithelioid leiomyosarcomas may show lesser degree of staining for these smooth muscle markers. Further, the high-grade tumor area also grossly expressed the progesterone and estrogen receptor, which can be expressed in up to $40 \%$ of cases of ULMS [3]. As evidenced in our case, there were multiple IHC features that supported a diagnosis of both ULMS and ESS. The high-grade sarcoma displayed mixed IHC pattern making it difficult to determine a specific entity.

A differential diagnosis for a uterine sarcoma that contains epithelial features may also include carcinosarcoma, which was originally classified as a uterine sarcoma by the 1998 FIGO classification system. Carcinosarcomas are defined as having both malignant sarcomatous and malignant epithelial components within the same tumor. However, diagnosis of carcinosarcoma in this case is unlikely due to the two isolated tumor areas displaying distinct morphologic and immunophenotypes.

In this case, the metastatic disease displayed distinct components of poorly differentiated adenocarcinoma (C7 negative, CK20 positive) with focal signet ring cell features favoring an intestinal origin. The primary site for this suspected malignancy most commonly originates from the appendix or upper gastrointestinal system [13]. Imaging studies and endoscopies did not ascertain a gastrointestinal origin in this patient, who additionally has had a prior appendectomy. Although signet ring cells features are strong associated with malignancies of the gastrointestinal tract, a differential diagnosis of primary ovarian neoplasms cannot be excluded. Several cases of primary gynecological tumors containing signet ring cell features have been reported in the literature $[14,15]$. Although further lymph node sampling was not completed at the time of surgery, pathologic studies of the para-aortic and femoral lymph nodes could have supported our differential diagnosis of a metastatic versus primary neoplasm.

A review of the literature produced no similar cases of high grade uterine sarcoma colliding with a metastat- 
ic adenocarcinoma to the uterus. A PubMed search using the criteria "( (uterine sarcoma AND metastatic adenocarcinoma) AND case report)" resulted in 50 cases of uterine sarcomas, none of which presented with a second metastatic adenocarcinoma to the uterus. Further refining the search criteria to "uterine leiomyosarcoma" and/or "endometrial stroma sarcoma" resulted in fewer related cases.

In summary, uterine sarcoma is a rare cancer with an annual incidence of 0.36 per 100,000 woman-years [16]. Furthermore, the collision of a uterine sarcoma with a metastatic adenocarcinoma to the uterus has not yet been described in the literature. Thus, we report a rare case of high grade uterine sarcoma colliding with metastatic poorly differentiated adenocarcinoma with focal signet ring cell features.

\section{Consent}

Written informed consent was obtained from the patient for publication of this case report and accompanying images. A copy of the written consent is available for review by the Editor-in-Chief of this journal on request.

\section{Sources of Support}

None.

\section{References}

1. Howlader N, Noone AM, Krapcho M, Miller D, Bishop K, et al. (2017) SEER Cancer Statistic Review, 1975-2014. National Cancer Institute, Bethesdam, USA.

2. Major FJ, Blessing JA, Silverberg SG, Morrow CP, Creasman WT, et al. (1993) Prognostic factors in early stage uterine sarcoma: A gynecologic oncology group study. Cancer 71: 1702-1709.

3. D'Angelo E, Prat J (2009) Uterine sarcomas: A review. Gynecol Oncol 116: 131-139.

4. Echt G, Jepson J, Steel J, Langholz B, Luxton G, et al. (1990) Treatment of uterine sarcomas. Cancer 66: 35-39.

5. Stanley R Hamilton, Lauri A Aaltonen (2003) World Health Organization classification of tumours. In: Tavassoli FA,
Devilee $P$, Pathology and genetics of tumours of the breast and female genital organs. IARC Press, Lyon.

6. Friedman CF, Hensley ML (2018) Options for adjuvant therapy for uterine leiomyosarcoma. Curr Treat Options Oncol 19: 7.

7. Rauh-Hain JA, Hinchcliff EM, Oduyebo T, Worley MJ Jr, Andrade CA, et al. (2014) Clinical outcomes of women with recurrent or persistent uterine leiomyosarcoma. Int J Gynecol Cancer 24: 1434-1440.

8. Chan JK, Kawar NM, Shin JY, Osann K, Chen LM, et al. (2008) Endometrial stromal sarcoma: A population-based analysis. Br J Cancer 99: 1210-1215.

9. Rauh-Hain JA, del Carmen MG (2013) Endometrial stromal sarcoma: A systematic review. Obstet Gynecol 122: 676683.

10. D’Angelo E, Spangoli LG, Prat J (2009) Comparative clinicopathologic and immunohistochemical analysis of uterine sarcomas diagnosed using the World Health Organization classification system. Human Pathol 40: 1571-1585.

11. Tse KY, Crawford R, Ngan H (2011) Staging of uterine sarcomas. Best Practice \& Research Clinical Obstetrics \& Gynaecology 25: 733-749.

12. Chiang S, Lee $\mathrm{CH}$, Stewart CJ, Oliva E, Hoang LN, et al. (2017) BCOR is a robust diagnostic immunohistochemical marker of genetically diverse high-grade endometrila stroma sarcoma, including tumors exhibiting variant morphology. Mod Pathol 30: 1251-1261.

13. Hou Y, Li Y, Shen D, Cui H (2011) Primary peritoneal serous carcinoma with signet ring cells. Gynecol Oncol Case Rep 1: 4-5.

14. Ganesh PG, Chander VR, Kanchana MP, Narasimhan L (2014) Primary ovarian mucinous carcinoma with signet ring cells- report of a rare case. J Clin Diagn Res 8: 12-13.

15. Boyd C, Cameron I, McCluggage WG (2010) Endometrila adenocarcinoma with signet ring cells: Report of two cases of an extremely rare phenomenon. Int J Gynecol Pathol 29: 579-582.

16. Koivisto-Korander R, Martinsen JI, Weidepass E, Leminen A, Pukkala E (2012) Incidence of uterine leiomyosarcoma and endometrial stromal sarcoma in Nordic countries: Results from NORDCAN and NOCCA databases. Maturitas 72: $56-60$. 